\title{
Triglyceride Concentration is Independently Associated with Variation in the LPL Gene in African American and European American Women
}

\author{
Krista Casazza ${ }^{*}, 1$, Nihal Natour ${ }^{1}$, Jasmin Divers ${ }^{5}$, Laura Kelly Vaughan ${ }^{2}$, Abigail W. Bigham ${ }^{4}$, \\ Barbara A. Gower ${ }^{1}$, Gary R. Hunter ${ }^{1,3}$ and José R. Fernández ${ }^{1,2}$
}

${ }^{I}$ Department of Nutrition Sciences and Clinical Nutrition Research Center, ${ }^{2}$ Section on Statistical Genetics, Department of Biostatistics, and ${ }^{3}$ Department of Human Studies, University of Alabama at Birmingham, Birmingham, AL; ${ }^{4}$ Department of Anthropology, The Pennsylvania State University, University Park, PA; ${ }^{5}$ Section on Statistical Genetics and Bioinformatics, Department of Public Health Sciences, Wake Forest University School of Medicine, Winston-Salem, $N C, U S A$

\begin{abstract}
Objective: The regulation of triglyceride (TG) metabolism may be affected by many factors including: race/ethnicity, body composition, body fat distribution, insulin action, and the activity of the lipoprotein lipase (LPL) enzyme. This study was designed to identify the independent effects of body composition, body fat distribution, insulin action, and LPL genetic variation on TG and HDL-C concentration. Subjects: The study consisted of premenopausal European American (EA) ( $\mathrm{n}=114)$ and African American (AA) $(\mathrm{n}=140)$ women. Measurements: Body composition was measured by dual-energy X-ray absorptiometry (DXA), intra-abdominal adipose tissue (IAAT) by computed tomography (CT), insulin sensitivity (SI) and the acute insulin response to glucose (AIRg) by intravenous glucose tolerance test, median household income by zip-code. An estimate of individual genetic admixture was obtained from the genotyping of 85 ancestry informative markers and used to control for population stratification. Multiple linear regression analyses were used to identify contributions of body composition, IAAT, SI, AIRg, African genetic admixture, and LPL polymorphisms to TG and HDL-C. Results: AA had lower TG and higher HDL-C than EA. African genetic admixture, IAAT, AIRg, SI, and RS285 were significantly and independently related to TG concentration. SI was the only independent contributor to HDL-C. RS1800590 was not associated with variation in TG or HDL. Conclusion: Triglyceride concentration appears to be multifactorial in origin and emanates in part from variance in LPL RS285. Future investigations are necessary to understand the mechanisms through which gene polymorphisms, body fat distribution, and insulin action influence the lipid profile.
\end{abstract}

Keywords: Triglyceride, HDL-C, LPL, premenopausal women, gene polymorphism, insulin sensitivity.

\section{INTRODUCTION}

The regulation of triglyceride (TG) metabolism is affected by many factors (e.g., body composition, body fat distribution, insulin action, and the activity of the lipoprotein lipase (LPL) enzyme). Adverse lipid profiles may influence obesity, type 2 diabetes, and cardiovascular disease (CVD) [1] and genetic differences in the regulation of TG may involve different contributing factors. AA females, who have a higher risk for the aforementioned chronic diseases are known to have lower TG concentration and higher high density lipoprotein cholesterol (HDL-C) than their EA counterparts [2]. It is plausible that genetic or physiological differences in factors involved in lipid metabolism could account for differences in lipid profile among EA and AA.

Genetic differences in the regulation of lipids may be due to polymorphisms in the lipoprotein lipase (LPL) gene (located on chromosome 8p22) [3-5]. LPL is a key enzyme involved in the process of lipolysis and functions by

*Address correspondence to this author at the Department of Nutrition Sciences, Webb 415, $15303^{\text {rd }}$ Ave S, University of Alabama at Birmingham, Birmingham, AL 35294-3360, USA; Tel: (205) 975-6226; Fax: (205) 934-7050; E-mail: kristac@uab.edu releasing fatty acids from the cores of chylomicrons and VLDL. These NEFA are subsequently oxidized or reesterified into TG within adipose tissue, liver, or other tissues. Individual differences in LPL activity may be reflected in concentration of circulating $\mathrm{TG}$ or tissue deposition of TG. Differences in LPL activity may also be reflected in the circulating concentration of HDL-C as alteration in TG hydrolysis influences the production of many remnants necessary for the synthesis of HDL-C $[6,7]$.

Several polymorphisms in the LPL gene including the D9N, N291S, S447X, RS1800590, and RS285 mutations [5, 8] have been proposed as key players in the regulation of lipids in different populations. This study focuses on RS285 (495C/T) and RS1800590 (93T/G), two polymorphisms that differ in frequency between AA and EA populations and are known to affect TG and HDL-C levels. For instance, the T allele of LPL RS285 has been shown to be present in $~ 95 \%$ of individuals of African ancestry, but only occurs in $~ 50 \%$ of individuals of European ancestry [8,9]. Similarly, the G allele of LPL RS1800590 has been observed at a rate of $\sim 1.5 \%$ [8] in Europeans and $76.4 \%$ in Africans [10]. The influence of the LPL RS1800590 and RS285 polymorphisms on the lipid profile is not clear. In several studies, the T allele of RS285 and the G allele of RS1800590 were associated 
with lower TG and higher HDL-C [9-13]. In contrast, other studies show no relationship between LPL genotype and the lipid profile [14-16]. The discrepancies among study findings may be due to population differences i.e. differences may only arise when studies are conducted in a sample of non-homogeneous individuals [17]. It is possible that the effect of LPL genotype on the lipid profile differs with race/ethnicity, such that an effect may be observed in a population with a greater proportion of one genetic background, but absent in a population with a greater proportion of another genetic background or if the allele frequencies in one population is too low to detect a difference.

Fat distribution and insulin action have also been shown to influence the lipid profile. In particular, intra-abdominal adipose tissue (IAAT) may be a unique pathogenic fat depot because it secretes substances (adipocytokines, vasoactive) that can adversely influence TG and HDL-C metabolism [18]. A causal relationship has been established between hyperinsulinemia and hypertriglyceridemia [19] generally associating hyperinsulinemia with elevated TG (via the effects on lipoprotein metabolism and regulation of LPL activity) [20]. However, this association between TG and insulin differs by racial/ethnic groups, with AA shown to have higher fasting insulin, higher AIRg and lower insulin sensitivity (SI) in the presence of lower TG [19]. This may suggest that hypertriglyceridemia reflects insulin resistance in individuals of European ancestry, but not those of African origin.

This study was designed to identify the independent effects of body composition, body fat distribution, insulin response and action, and LPL polymorphisms on circulating concentrations of TG and HDL-C within a bi-ethnic population of healthy premenopausal women. Responding to the concerns of inflated Type I error rates in association studies due to population stratification [21], and to account for the role that ancestral background might have on differences in TG and HDL-C among populations, a measure of genetic admixture was obtained for each individual and used as a genomic control factor when testing the association between the LPL gene polymorphisms and levels of TG and HDL-C.

\section{EXPERIMENTAL METHODS}

Data were evaluated from 254 women who participated in two ongoing studies at the University of Alabama at Birmingham (UAB) evaluating the role of diet and exercise in metabolic outcomes. These studies are described in greater detail elsewhere [22]. Subjects were normoglycemic premenopausal AA $(n=140)$ and EA $(n=114)$ women aged 20-41 years with sedentary lifestyles and no history of diabetes. In addition, all subjects were nonsmokers and were not taking any medications known to affect body composition, energy expenditure, insulin level, heart rate, lipid metabolism, or thyroid function. Body mass index (BMI) ranged from 21 to $31 \mathrm{~kg} / \mathrm{m}^{2}$. For racial classification, subjects identified themselves, their parents, and their grandparents as either AA or EA. All participants were informed of the study design and objectives and informed consent was obtained. The Institutional Review Board at University of Alabama at Birmingham (UAB) approved the protocols for both studies.
Participants were admitted to the General Clinical Research Center (GCRC) as inpatients for $\sim 72$ hrs, during which metabolic testing was conducted as described below.

\section{Body Composition and Fat Distribution}

Body composition (total fat mass) was measured by dualenergy X-ray Absorptiometry (DXA) using either a Lunar DPX-L densitometer (LUNAR Radiation Corp., Madison, WI) or a LUNAR Prodigy densitometer in the Department of Nutrition Sciences at UAB as previously described [22, 23]. We have shown in our laboratory that body composition assessed by these instruments generally differs by $4 \%$ or less [24]. Subjects were scanned in light clothing while lying flat on their backs with arms at their sides. Intra-abdominal adipose tissue (IAAT) was measured by computed tomography (CT) scanning with a HiLight/Advantage Scanner (General Electric, Milwaukee) as previously described [25]. We have shown the test-retest reliability for IAAT to be 1.7 percent [26].

\section{Collection of Sera and Frequently-Sampled, Intravenous, Glucose Tolerance Test (FSIGT)}

At approximately 7: 00 am, after a 12-hour fast, flexible intravenous catheters were placed in the antecubital spaces of both arms as described elsewhere [23]. Three blood samples were drawn over a 40-min period and sera was subsequently separated and pooled for analysis of lipids. Three additional blood samples were taken over a 20 -min period for determination of basal glucose and insulin (the average of the values was used for basal "fasting" concentrations). At time " 0 ", glucose (50\% dextrose; 11.4 $\mathrm{g} / \mathrm{m}^{2}$ ) was administered intravenously. At minute 20 following glucose administration, subjects received an intravenous bolus of either tolbutamide $\left(125 \mathrm{mg} / \mathrm{m}^{2}\right)$ or insulin (0.02 units $/ \mathrm{kg})$. Because SI results from tolbutamidemodified tests have been shown to be approximately $16 \%$ higher than those from insulin-modified tests [27], adjustments by tests type were evaluated as discussed in the statistical analyses. Sera were analyzed for glucose and insulin, and values were entered into the MINMOD computer program (version 3.0, (C) Richard N. Bergman) for determination of the insulin sensitivity index (SI) and the acute insulin response to glucose $\left(\mathrm{AIR}_{\mathrm{g}}\right)$ [28-30]. $\mathrm{AIR}_{\mathrm{g}}$ is the integrated incremental area under the curve for insulin during the first 10 minutes of the test.

\section{Assay of Glucose, Insulin, and Lipids}

Analyses were performed in the Core Laboratory of the GCRC and the Clinical Nutrition Research Center at UAB. Glucose was measured in $10 \mu \mathrm{l}$ sera using an Ektachem DT II System (Johnson and Johnson Clinical Diagnostics). In the Core Laboratory, this analysis has a mean intra-assay coefficient of variation (c.v.) of $0.61 \%$, and a mean interassay c.v. of $1.45 \%$. For a portion of the women, insulin was assayed in duplicate $200 \mu \mathrm{l}$ aliquots with Diagnostic Products Corporation (Los Angeles, CA) "Coat-A-Count" kits. In the Core Laboratory, this assay has a sensitivity of $1.9 \mu \mathrm{IU} / \mathrm{ml}$, a mean intra-assay c.v. of $5 \%$, and a mean interassay c.v. of $6 \%$. For the remainder of the women, insulin 
was assayed in duplicate $100 \mu \mathrm{l}$ aliquots with Linco Research Products Inc. (St. Charles, MO) reagents. In the Core Laboratory, this assay has a sensitivity of $3.35 \mu \mathrm{IU} / \mathrm{ml}$, a mean intra-assay c.v. of $3.49 \%$, and a mean interassay c.v. of $5.57 \%$. Commercial quality control sera of low, medium, and high insulin concentration are included in every assay to monitor variation over time. HDL-cholesterol (HDL-C) and triaclyglcerol (TG) were measured with the Ektachem DT II System. With this system, HDL-C is measured after precipitation of low-density lipoprotein cholesterol (LDL-C) and very-low-density lipoprotein cholesterol with dextran sulfate and magnesium chloride. Control sera of low and high substrate concentration are analyzed with each group of samples, and values for these controls must fall within accepted ranges before samples are analyzed. The DT II is calibrated every six months with reagents supplied by the manufacturer.

\section{Genotyping}

Genotyping for the LPL RS1800590 and RS285 polymorphisms were performed at The Pennsylvania State University. The LPL RS285 mutation occurs at intron 6 in the LPL gene and involves a $\mathrm{C} \rightarrow \mathrm{T}$ transition that causes the deletion of the cut site for the restriction enzyme Pvu II. The LPL RS1800590 polymorphism involves $\mathrm{T} \rightarrow \mathrm{G}$ transmission at nucleotide -93 of the LPL promoter. For the genotyping of both mutations, polymerase chain reaction (PCR) amplification of genomic DNA was performed as described elsewhere [31].

Genotyping of the ancestry informative markers (AIMs) for the measurement of genetic admixture was performed at Prevention Genetics (www.preventiongenetics.com) using the Chemicon Amplifuor SNPs Genotyping System (Genome Research 11: 163-169, 2001) coupled with ArrayTape technology (www.global-array.com). A panel of 85 ancestry informative markers was used to estimate the genetic admixture proportion of each subject. Molecular techniques for the allelic identification and methodology for genetic admixture application have been described elsewhere [32] and information regarding marker sequences, experimental details, and parental population allele frequencies has been submitted to dbSNP (http: //www.ncbi.nlm.nih.gov/SNP/) under the handle PSU-ANTH. The information from the AIMs was translated into estimates of African (AFADM) and European admixture (EUADM) for each subject using AdmixMap software [33]. Admixture estimates ranged from $1-100$, for a total of 100 on each subject.

\section{Socioeconomic Status}

Median household income according to zip code of subject's residence was used as a surrogate for socioeconomic status. Information about median income was obtained from the US census (http: //factfinder.census.gov/ servlet/ SAFFFacts). Geographic differences in health status, based on neighborhood characteristics are well documented. Although zip code boundaries are not perfect measures in assessing SES of neighborhoods, they do represent local residential areas and they are frequently used as an assessment of neighborhood SES [34].

\section{Statistical Analysis}

To evaluate possible confounding effects of different protocols used on the two studies for the measurement of FSIGT, insulin assay type, and body composition betweenstudy differences were assessed by three exploratory methods. In the first one, the entire sample was divided according to study and exploratory regression models were evaluated according to study. In addition, for the measures of concern, individual values for each subject within a study were transformed into "Z-scores" and these scores were subsequently used for exploratory analyses. Also, raw data were statistically adjusted for measurement type and evaluated in exploratory models using the entire sample. Same exploratory results were obtained when the regression models were ran by study. Although in all exploratory models no significant differences or contributions were assessed between the studies, between-study differences were accounted for statistically by including a variable for test type method in all analyses, accounting for the possible confounding of residual variance.

Descriptive statistics were obtained for variables of interest. DNA and/or insulin measures were not available on all subjects. There were no differences in demographics between those that had all information and those that did not. Including only subjects who were informative for all variables $(n=184 ; A A=97 ; E A=87)$, two multiple regression models were used to test for the independent contributions to TG and HDL-C; the first model (non-genetic) included body composition, fat distribution, insulin response and action, and the second (genetic) included genetic polymorphisms, after adjusting for genetic admixture. For the non-genetic models testing for the dependent variable $\mathrm{TG}$, the independent variables SI, $\mathrm{AIR}_{\mathrm{g}}$, SES, total fat and IAAT were included as predictors. For the dependent variable HDL-C AIRg was replaced by TG. For the model with genetic contributors, three genetic models were evaluated during preliminary data analysis: an allelic model testing the presence or absence of the mutant allele (equivalent to a dominant genetic model), a genotypic model (equivalent to an additive genetic model) testing the three possible combinations of alleles, and a recessive model. In the final analysis, only the allelic model was used because the genotypic and recessive models did not explain phenotypic variability.

To conform to the assumptions of linear regression, all statistical models were evaluated for residual normality, constant variance and outliers, and logarithmic transformations were performed when appropriate. Those residuals that deviated above and below three standard deviations were removed in the final models. All data was analyzed using SAS 9.1 software.

\section{Statement of Ethics}

We certify that all applicable Institutional Review Board regulations concerning the ethical use of human volunteers were followed during this research

\section{RESULTS}

Descriptive statistics for the total study group $(n=254)$, and according to race $(\mathrm{EA}=114, \mathrm{AA}=140)$, are listed in 
Table 1. Descriptive Statistics of the Study Subjects

\begin{tabular}{|c|c|c|c|}
\hline \multirow[b]{2}{*}{ Variable } & \multirow{2}{*}{$\begin{array}{c}\text { Total } \\
\text { Mean } \pm \text { SD }\end{array}$} & \multicolumn{2}{|c|}{ RACE } \\
\hline & & $\begin{array}{c}\text { AA } \\
\text { Mean } \pm \text { SD }\end{array}$ & $\begin{array}{c}\text { EA } \\
\text { Mean } \pm \text { SD }\end{array}$ \\
\hline Age (year) & $34.0 \pm 5.9$ & $33.6 \pm 5.7$ & $34.5 \pm 6.2$ \\
\hline $\operatorname{AIR}_{\mathrm{g}}(\mu \mathrm{IU} / \mathrm{ml} \times 10)$ & $754.2 \pm 544$ & $950.3 \pm 611.5$ & $530.0 \pm 337.9^{* * *}$ \\
\hline $\mathrm{SI}\left(\times 10^{-4} \mathrm{~min}^{-1} /(\mu \mathrm{lU} / \mathrm{ml})\right)$ & $3.6 \pm 2.5$ & $3.3 \pm 2.6$ & $4.0 \pm 2.3^{*}$ \\
\hline BMI & $27.5+2.3$ & $27.4+2.5$ & $27.7+2.2$ \\
\hline Total fat (kg) & $31.2 \pm 6.7$ & $30.2 \pm 6.6$ & $32.3 \pm 6.6^{*}$ \\
\hline Percent body fat & $42.0+5.3$ & $41.4+5.7$ & $42.8+4.7^{*}$ \\
\hline $\operatorname{IAAT}\left(\mathrm{cm}^{2}\right)$ & $75.9 \pm 31.9$ & $63.3 \pm 26.9$ & $90.8 \pm 31 * * *$ \\
\hline $\mathrm{TG}(\mathrm{mg} / \mathrm{dl})$ & $88.8 \pm 47.9$ & $68.7 \pm 26.3$ & $113.6 \pm 56.3 * * *$ \\
\hline HDL-C (mg/dl) & $40.3 \pm 10.6$ & $43.4 \pm 10.8$ & $36.6 \pm 9 * * *$ \\
\hline Income (dollars) & $43675 \pm 16550$ & $37613 \pm 14579$ & $50747 \pm 15956^{* * *}$ \\
\hline AFADM & $0.47 \pm 0.29$ & $0.71 \pm 0.1$ & $0.17 \pm 0.06^{* * *}$ \\
\hline
\end{tabular}

${ }^{*} p<0.05$. ${ }^{* *} p<0.01 .{ }^{* * *} p<0.001$ AA vs EA. AIR ${ }_{\mathrm{g}}=$ acute insulin response to glucose; SI= insulin sensitivity; BMI= body mass index; IAAT= intraabdominal adipose tissue; $\mathrm{TG}=$ triaclyglcerol concentration; $\mathrm{HDL}-\mathrm{C}=$ high density lipoprotein cholesterol; $\mathrm{AFADM}=\mathrm{African}$ admixture.

Table 1. There were significant differences between EA and AA with respect to insulin response, lipid profile, and body composition, with AA having higher AIR and HDL-C and EA having greater SI, total fat mass, IAAT, and TG. EA also had a higher median income than AA and therefore this proxy measure of SES was included in all analyses.

\section{Hardy-Weinberg Equilibrium}

RS285 was not in Hardy-Weinberg equilibrium (HWE) in the total sample $\left(\chi^{2}=6.5962, p=0.0102\right)$, but was in HWE when stratified by race $\left(\chi_{\mathrm{EA}}^{2}=0.0007, \mathrm{p}=0.9788 ; \chi_{\mathrm{AA}}^{2}=2581\right.$, $\mathrm{p}=0.6114)$. RS1800590 was not in HWE in the total sample $\left(\chi^{2}=13.091, \mathrm{p}=0.0002\right)$ or when considering EA $\left(\chi^{2}=8.7793\right.$, $\mathrm{p}=0.0030)$ separately, but was in HWE among $\mathrm{AA}\left(\chi^{2}=5861\right.$, $\mathrm{p}=0.4439)$. Collectively, these results suggest that the deviation from HWE was likely due to population structure [21].

\section{LPL Polymorphisms}

The genotypic frequency of the LPL RS285 polymorphism for the entire group was $50 \%, 36 \%$, and $14 \%$ for TT, CT, and CC genotypes, respectively (Table 2). Significant differences in the frequency of the polymorphism existed between AA and EA individuals. The frequency of the T allele was greater in AA than in EA women; $\left(\chi^{2}=32.5\right.$, $\mathrm{p}<0.001)$. In addition, the carriers of the $\mathrm{T}$ allele had greater $\operatorname{AIR}_{\mathrm{g}}(\mathrm{p}<0.05)$ and AFADM $(\mathrm{p}<0.001)$, whereas non-carriers had a greater TG $(p<0.01)$ and EUADM $(p<0.001)$. The results support previous findings that allele $\mathrm{T}$ plays a role in lower levels of TG [9].

The genotypic frequency for LPL RS1800590 in the entire group was $62.3 \%, 27.4 \%$, and $10.2 \%$ for TT, GT, and GG genotypes, respectively (Table 2). Significant differences in the frequency of the polymorphism existed between AA and EA individuals. African Americans were more frequently carriers of the $G$ allele compared to EA;

Table 2. Genotype Frequency of LPL Polymorphisms in the Total Sample and According to Race

\begin{tabular}{|c|c|c|c|}
\hline Genotype & Total Sample & $\mathrm{AA}(\mathrm{n}=124)$ & EA $(n=91)$ \\
\hline \multicolumn{4}{|c|}{ LPL RS1800590 } \\
\hline $\mathrm{T} / \mathrm{T}$ & $134(62.3 \%)$ & 48 (38.7\%) & $86(94.5 \%)$ \\
\hline $\mathrm{T} / \mathrm{G}$ & $59(27.4 \%)$ & $55(44.4 \%)$ & $4(4.4 \%)$ \\
\hline $\mathrm{G} / \mathrm{G}$ & $22(10.2 \%)$ & $21(16.9 \%)$ & $1(1.1 \%)$ \\
\hline \multicolumn{4}{|c|}{ LPL RS285 } \\
\hline $\mathrm{C} / \mathrm{C}$ & $30(14 \%)$ & $3(2.4 \%)$ & $27(29.7 \%)$ \\
\hline $\mathrm{T} / \mathrm{C}$ & $77(36 \%)$ & $28(22.6 \%)$ & $49(53.8 \%)$ \\
\hline $\mathrm{T} / \mathrm{T}$ & $108(50 \%)$ & $93(75.0 \%)$ & $15(16.5 \%)$ \\
\hline
\end{tabular}


$\left(\chi^{2}=70, p<0.001\right)$. In addition, the carriers of the $\mathrm{G}$ allele had a greater $\operatorname{AIR}_{g}(\mathrm{p}<0.001)$ and AFADM $(\mathrm{p}<0.001)$ than noncarriers, whereas non-carriers had greater total fat $(\mathrm{p}<0.05)$, IAAT $(\mathrm{p}<0.001)$, and TG $(\mathrm{p}<0.001)$ and EUADM $(\mathrm{p}<0.001)$ than carriers.

\section{Predictors of Triglyceride and HDL-C Levels}

Multiple linear regression analyses were used to evaluate the independent effect of various factors on TG and HDL-C using a genetic and a non-genetic model. Table $\mathbf{3}$ presents results of the non-genetic model for the total sample and according to race. As shown in Table 3, in the total sample, IAAT was significantly and positively associated with TG such that increased IAAT was significantly related to increased TG. Conversely, AIRg was negatively associated with TG concentration, such that increased AIRg was related to lower TG. When results were analyzed according to race, IAAT was positively associated with TG among both EA and AA. However, SI was only associated with TG concentration in AA and exhibited an inverse relationship. Table $\mathbf{3}$ also presents the non-genetic results of independent predictors of HDL-C. The results indicated that in the total sample IAAT was a significant contributor, showing that IAAT was significantly and negatively associated with HDL-C. However, among the variables we identified as potential contributors, none were found to be significant predictors of HDL-C when EA were analyzed separately. In AA, higher HDL-C was associated with less IAAT and greater SI.
The LPL RS1800590 polymorphism was not associated with either TG or HDL concentration in any analysis. Table 4 presents results for the models that included genetic analysis including RS285. African genetic admixture was associated with TG concentration such that greater African genetic admixture was associated with lower TG $(\mathrm{P}<0.001)$, accounting for $\sim 21 \%$ of the variance in this model. African genetic admixture demonstrated a trend towards significance in the association with HDL $(\mathrm{p}=0.07)$ such that greater African admixture was associated with higher HDL. Similar to the results generated in the non-genetic model, higher TG concentration was associated with lower AIRg (accounting for $\sim 15 \%$ of variance) and higher IAAT (accounting for $30 \%$ of variance). SI ( 19\% of variance) and LPL RS285 ( $20 \%$ of variance) were also shown to be significant contributors to TG concentration such that elevated TG was associated with lower SI and the presence of the T allele of RS285. SI was the only significant predictor of HDL-C, although IAAT demonstrated a trend towards significance. The results remained significant after controlling for the effect of multiple comparisons via permutation tests $(1000$ simulations) to generate empirical $\mathrm{P}$ values under the null hypothesis of no association between genotype and/or admixture and traits (data not shown).

\section{DISCUSSION}

To our knowledge, this is the first study to evaluate the association of metabolic and genetic predictors of TG and

Table 3. Multiple Regression Models* Showing Independent Effects of Body Composition, Body Fat Distribution and Insulin Response and Action on the Dependent Variables TG and HDL within a Bi-Ethnic Population of Healthy Premenopausal Women

\begin{tabular}{|c|c|c|c|c|c|c|}
\hline \multicolumn{7}{|c|}{ TG } \\
\hline \multirow{2}{*}{ Model 1} & Total & \multirow{2}{*}{ p-value } & EA & \multirow{2}{*}{ p-value } & $\mathbf{A A}$ & \multirow{2}{*}{ p-value } \\
\hline & Parameter Estimate & & Parameter Estimate & & Parameter Estimate & \\
\hline Intercept & $4.00+0.63$ & $<0.001$ & $3.82+1.01$ & $<0.001$ & $4.73+0.78$ & $<0.001$ \\
\hline Income & $0.00+0.00$ & 0.07 & $0.00+0.00$ & 0.72 & $0.00+0.00$ & 0.81 \\
\hline Log AIRg & $-0.16+0.05$ & 0.002 & $-0.05+0.09$ & 0.60 & $-0.12+0.07$ & 0.10 \\
\hline Log SI & $-0.08+0.06$ & 0.19 & $-0.08+0.10$ & 0.41 & $-0.18+0.08$ & 0.03 \\
\hline LogTotal Fat & $-0.16+0.16$ & 0.34 & $-0.21+0.28$ & 0.45 & $-0.15+0.19$ & 0.43 \\
\hline Log IAAT & $0.42+0.07$ & $<0.001$ & $0.38+0.15$ & 0.01 & $0.21+0.10$ & 0.04 \\
\hline \multicolumn{7}{|c|}{ HDL } \\
\hline \multirow{2}{*}{ Model 1} & Total & \multirow{2}{*}{ p-value } & EA & \multirow{2}{*}{ p-value } & $\mathbf{A A}$ & \multirow{2}{*}{ p-value } \\
\hline & Parameter Estimate & & Parameter Estimate & & Parameter Estimate & \\
\hline Intercept & $4.35+0.32$ & $<0.001$ & $4.28+0.51$ & $<0.001$ & $4.01+0.52$ & $<0.001$ \\
\hline Income & $-0.00+0.00$ & 0.45 & $0.00+0.00$ & 0.64 & $-0.00+0.00$ & 0.45 \\
\hline $\log$ TG & $-0.06+0.04$ & 0.11 & $-0.02+0.05$ & 0.73 & $-0.06+0.07$ & 0.39 \\
\hline Log SI & $0.04+0.03$ & 0.25 & $0.01+0.05$ & 0.85 & $0.09+0.05$ & 0.05 \\
\hline LogTotal Fat & $0.09+0.09$ & 0.34 & $-0.02+0.15$ & 0.91 & $0.15+0.12$ & 0.24 \\
\hline Log IAAT & $-0.16+0.05$ & $<0.001$ & $-0.12+0.08$ & 0.13 & $-0.13+0.07$ & 0.04 \\
\hline
\end{tabular}

*Adjusted for test type.

$\mathrm{TG}=$ triaclyglcerol concentration; $\mathrm{IAAT}=$ intra-abdominal adipose tissue; $\mathrm{SI}=$ insulin sensitivity; $\mathrm{AIR}_{\mathrm{g}}=$ acute insulin response to glucose. 
Table 4. Multiple Regression Models for the Association of the LPL RS285 Polymorphism with Circulating Concentrations of TG and HDL in a Bi-Ethnic Population of Healthy Premenopausal Women. All Models were Adjusted for Test Type and by AFADM to Control for Population Stratification

\begin{tabular}{|c|c|c|c|c|c|}
\hline \multicolumn{3}{|c|}{ TG } & \multicolumn{3}{|c|}{ HDL } \\
\hline Model 2 & Parameter Estimate & p-value & Parameter & Estimate & p-value \\
\hline Intercept & $4.53+0.65$ & $<0.001$ & Intercept & $4.20+0.36$ & $<0.001$ \\
\hline Income & $0.00+0.00$ & 0.58 & Income & $-0.00+0.00$ & 0.10 \\
\hline $\log \mathrm{AIR}_{\mathrm{g}}$ & $-0.12+0.06$ & 0.04 & $\log \mathrm{TG}$ & $0.05+0.10$ & 0.62 \\
\hline Log SI & $-0.15+0.07$ & 0.03 & $\log \mathrm{SI}$ & $0.09+0.03$ & 0.04 \\
\hline Log IAAT & $0.32+0.10$ & 0.002 & Log IAAT & $-0.10+0.06$ & 0.07 \\
\hline LPL RS 285 & $-0.14+0.06$ & 0.02 & LPL RS 285 & $-0.02+0.03$ & 0.62 \\
\hline AFADM & $-0.05+0.14$ & $<0.001$ & AFADM & $0.15 \pm 0.08$ & 0.07 \\
\hline
\end{tabular}

$\mathrm{TG}=$ triaclyglcerol concentration; IAAT = intra-abdominal adipose tissue; $\mathrm{SI}=$ insulin sensitivity; AFADM=African genetic admixture.

HDL-C implementing a study design that controls for the effects of environmental parameters (SES) and ancestral genetic background. IAAT, AIRg, SI, and the LPL RS285 polymorphism were related to TG suggesting that these are among a number of factors influencing lipid levels. SI was the only significant contributor to HDL-C.

The association of LPL RS285 with TG suggests that variation in LPL RS285 or the presence of the T allele may have an influence on determination of TG concentration. We found a significant inverse association of SI with TG, which supports previously documented associations between insulin action and elevated TG levels. Our study design, which involved adjusting for genetic admixture provided validity to our findings, and expands previous findings demonstrating that, regardless of racial/ethnic categorization, LPL RS285 is related to TG concentration.

The negative contribution of $\mathrm{AIR}_{\mathrm{g}}$ to $\mathrm{TG}$ (after adjustment for SI) suggests that relatively higher levels of post-prandial insulin inhibits lipolysis, and the resultant decrease in influx of NEFA to the liver limits biosynthesis of TG as assessed in the fasted state. It has been suggested that greater AIRg contributes to lower TG among AA [35]. IAAT also was a significant independent contributor to TG, as observed in other populations [36-39]. Increased accumulation of IAAT has been linked to a number of adverse metabolic outcomes including hypertriglyceridemia and hyperinsulinemia, suggesting that the lower IAAT may lead to lower TG among AA. However, in our genetic analyses, RS285 remained a significant predictor of TG even when IAAT and insulin action were included in the model, supporting the notion that factors genetic as well as biological differences could be involved in lower TG among AA. This finding also supports the previous findings of Ehrenborg and colleagues [9] who observed the RS285 polymorphism was associated with TG concentration among European and African individual as well as the work of others carried out in other populations [10-13].

It appears likely that both genetic and physiological differences among AA and EA are key factors involved in ethnic/racial differences in lipid profile between EA and AA. Results of this study indicate that the presence of the $\mathrm{T}$ allele reduces TG and because the frequency of the allele is greater in AA, RS285 may play a role in lower TG among AA. The significant association between AFADM and TG also supports this view. In addition, the notion that hypertriglyceridemia reflects insulin resistance in individuals of European ancestry, but not those of African origin has been explored by Sumner et al., (2005) who observed that unlike is the case in individuals not of European origin, among those of AA origin, TG are cleared from the circulation even in the presence of insulin resistance, maintaining plasma TG concentration. This suggests only a weak association between TG levels and insulin resistance in African Americans. It is also plausible this limited association between TG and insulin is also reflected in HDL-C levels in AA. As such, Sumner et al conclude higher HDL-C in AA is due to lower TG and may not be influenced by insulin dynamics. Although we demonstrate a relationship between SI and TG in AA, the relationship is inverse and counterintuitive to what has been demonstrated among EA. As such the relationship may reflect multi co-linearity between race and insulin dynamics in this sample. Nevertheless, our finding support both genetic and inherent physiological differences between EA and AA affect lipid metabolism.

Similar to research previously reported $[6,10,16]$, in the non-genetic model, IAAT was inversely associated with HDL-C (Table 3). With the inclusion of the genetic polymorphisms and adjustment for admixture, the inverse association with IAAT was reduced to a trend towards significance. It has been previously suggested that the influence of IAAT on HDL-C is mediated by either TG or insulin sensitivity $[23,40]$. In our model, SI was the only predictor related to HDL-C when all other factors were controlled for. This observation may suggest that higher SI leads to an elevation in HDL-C. Although the exact mechanism for this association is not clear, it has been suggested that hyperinsulinemia affects HDL clearance by depressing hepatic lipase activity and may be involved in this process $[5,41]$. Our data did not support a relationship between LPL polymorphisms and HDL-C, which coincides with other research reporting no association between LPL markers and HDL-C levels in humans [8, 10, 11, 13]. It is likely that any influence of LPL genotype on HDL-C is 
mediated via TG. Previous literature exploring the relationship between genetic admixture and HDL-C is limited; however, the possibility that LPL polymorphisms indirectly contribute to HDL-C levels by acting through other factors is plausible, particularly when taking into account previous associations of genetic admixture with both IAAT and SI $[42,43]$.

In contrast to work completed by other researchers [813], our results did not support an association between LPL RS1800590 and TG or HDL concentration. The inconsistency between our results and that of others may be explained by the inclusion of the D9N haplotype in studies by others evaluating effect of RS1800590 in many of those studies. In people of European ancestry, the G allele of LPL RS1800590 cosegregates with the variant allele A of the LPL polymorphism D9N, forming the $-93 \mathrm{G} / 9 \mathrm{~N}$ haplotype. This haplotype creates a "synergetic" relationship that results in overall higher levels of TG. In African descendents, there is no evidence of linkage disequilibrium between the two LPL variants [44]. Therefore in this bi-ethnic population, the haplotype was not studied. The independent effect of the LPL RS1800590 on TG levels in African descendents (where no linkage disequilibrium occurs with $\mathrm{D} 9 \mathrm{~N}$ ) has been previously studied, showing contradictory results [9, 10, 44]. The $G$ allele has been shown to have a TG lowering effect but no effect on HDL-C in some studies [9, 10, 44]. The lack of association between TG and HDL concentration in our sample is not readily apparent.

There were deviations from HWE for both polymorphisms. The deviations were likely due to population structure. In the RS285 polymorphism, the deviation noted in the total sample was likely a product of different frequencies between the populations. However, in RS1800590 the deviation in EA was likely due to the under-representation of heterozygotes. As such, the frequencies among EA likely drove the results in the total sample. Extensive quality control measures revealed that the deviation was not due to genotyping errors or other biases, but instead was a product of population structure. Controlling for admixture has been described as a valid method to adjust for deviations from HWE related to population stratification, and to decrease the chance of a false-positive association [21, 45]. Furthermore, controlling for AFADM, increases the likelihood that the observed association of AIRg, SI, IAAT and RS285 with TG reflect valid contributions to the variance, independent of race/ethnicity (Table $\mathbf{4}$ ).

This study was designed to include multiple physiologic and genetic variables measured using robust methodology that may contribute to the lipid profile. However, the study was limited by the relatively small number of subjects and the BMI range of inclusion. Also, only two polymorphisms for LPL were considered; other polymorphisms within the LPL gene may have contributed to the phenotypes of interests. The use of zip code median income as an index of socioeconomic status was a limitation, as it does not take into account the variability of social and financial factors that might occur within a zip code area.

In conclusion, these observations suggest that circulating TG concentrations reflect a complex interplay of genetic and physiologic factors including variance at the LPL RS285 gene. Further, differences in HDL-C may be due primarily to lower TG concentration. This study is the first to examine the relationships of TG and HDL-C with physiological and genetic variation within a comprehensive framework that accounts for genetic, environmental, and physiologic factors. Our results using the multiple linear regression approach allowed for the simultaneous examination of independent contributions of the polymorphisms, body composition, fat distribution and insulin dynamics. The fact that body fat distribution, insulin dynamics, and the LPL RS285 polymorphism independently contributed to TG concen-tration is strengthened by the inclusion of African genetic admixture as a covariate. The vast majority of the literature to determine the etiology of racial/ethnic differences in lipid profile relies on individual's self-classification of racial/ ethnic group. One of the concerns of genetic association studies is the confounding effect due to racial categorization and their contribution to the etiology of a disease. By using genetic admixture, we typified the genetic factors underlying racial classification and their contribution, if any, to the genotype-phenotype associations. Future investigations are necessary to fully understand the mechanisms through which gene polymorphisms, body fat distribution, and insulin response and action influence the lipid profile and how the associations differ according to ancestral genetic background.

\section{ACKNOWLEDGEMENTS}

JRF conceived the study, participated in its design and coordination, carried out data analysis, and contributed to the writing of the manuscript. KC, JD, LKV, and NN carried out the statistical analyses and contributed to the writing of the manuscript. AB carried out the genotyping and contributed to the genetic statistical analyses. BG and GH contributed to design and acquisition of human data. $\mathrm{BG}, \mathrm{KC}$, and JRF critically revised the manuscript. Additionally, none of the authors have potential conflicts of interest that could raise questions about a paper's credibility if disclosed later. Further, each author listed on the manuscript has seen and approved the submission of this version of the manuscript and takes full responsibility for the manuscript.

The authors wish to acknowledge Maryellen Williams and Cindy Zeng for laboratory analyses; Paul Zuckerman for study coordination; Scott Butsch and Greg Batson for physician coverage; David Bryan and Robert Petri for technical assistance; the help of the GCRC staff; and Nestlé Food Co., Solon, OH (Stouffer's Lean Cuisine) and HJ Heinz Co., Pittsburgh, PA (Smart Ones) for providing food.

Funded by: R01DK49779, M01-RR-00032, P30-DK56336.

\section{CONFLICT OF INTEREST}

This research manuscript and the data presented in this paper have not previously been published, either in whole or in part, and no similar paper is in press or under review elsewhere. The work is not and will not be submitted to any other journal while under consideration by The Open Obesity Journal. Additionally, none of the authors have potential conflicts of interest that could raise questions about a paper's credibility if disclosed later. Further, each author listed on the manuscript has seen and approved the 
submission of this version of the manuscript and takes full responsibility for the manuscript.

\section{REFERENCES}

[1] Mead JR, Irvine SA, Ramji DP. Lipoprotein lipase: structure, function, regulation, and role in disease. J Mol Med 2002; 80(12): 753-69.

[2] Sumner AE, Cowie CC. Ethnic differences in the ability of triglyceride levels to identify insulin resistance. Atherosclerosis 2008; 196(2): 696-703.

[3] Fisher RM, Humphries SE, Talmud PJ. Common variation in the lipoprotein lipase gene: effects on plasma lipids and risk of atherosclerosis. Atherosclerosis 1997; 135(2): 145-59.

[4] Goldberg IJ. Lipoprotein lipase and lipolysis: central roles in lipoprotein metabolism and atherogenesis. J Lipid Res 1996; 37(4): 693-707.

[5] Murthy V, Julien P, Gagne C. Molecular pathobiology of the human lipoprotein lipase gene. Pharmacol Ther 1996; 70(2): 10135

[6] Lamarche B, Rashid S, Lewis GF. HDL metabolism in hypertriglyceridemic states: an overview. Clin Chim Acta 1999; 286(1-2): 14561.

[7] Merkel M, Eckel RH, Goldberg IJ. Lipoprotein lipase: genetics, lipid uptake, and regulation. J Lipid Res 2002; 43(12): 1997-2006.

[8] Sagoo GS, Tatt I, Salanti G, et al. Seven lipoprotein lipase gene polymorphisms, lipid fractions, and coronary disease: a HuGE association review and meta-analysis. Am J Epidemiol 2008; 168(11): 1233-46.

[9] Ehrenborg E, Clee SM, Pimstone SN, et al. Ethnic variation and in vivo effects of the $-93 \mathrm{t}->\mathrm{g}$ promoter variant in the lipoprotein lipase gene. Arterioscler Thromb Vasc Biol 1997; 17(11): 2672-8.

[10] Talmud PJ, Hall S, Holleran S, et al. LPL promoter -93T/G transition influences fasting and postprandial plasma triglycerides response in African-Americans and Hispanics. J Lipid Res 1998; 39(6): 1189-96.

[11] Chamberlain JC, Thorn JA, Oka K, et al. DNA polymorphisms at the lipoprotein lipase gene: associations in normal and hypertriglyceridaemic subjects. Atherosclerosis 1989; 79(1): 85-91.

[12] Pasalic D, Sertic J, Kunovic B, et al. Lipoprotein lipase gene polymorphism and lipid profile in patients with hypertriglyceridemia. Croat Med J 2001; 42(5): 517-22.

[13] Wang XL, McCredie RM, Wilcken DE. Common DNA polymorphisms at the lipoprotein lipase gene. Association with severity of coronary artery disease and diabetes. Circulation 1996; 93(7): 1339-45.

[14] Hall S, Talmud PJ, Cook DG, et al. Frequency and allelic association of common variants in the lipoprotein lipase gene in different ethnic groups: the Wandsworth Heart and Stroke Study. Genet Epidemiol 2000; 18(3): 203-16.

[15] Lee WJ, Sheu WH, Jeng CY, et al. Associations between lipoprotein lipase gene polymorphisms and insulin resistance in coronary heart disease. Zhonghua Yi Xue Za Zhi (Taipei) 2000; 63(7): 563-72.

[16] Peacock RE, Hamsten A, Nilsson-Ehle P, et al. Associations between lipoprotein lipase gene polymorphisms and plasma correlations of lipids, lipoproteins and lipase activities in young myocardial infarction survivors and age-matched healthy individuals from Sweden. Atherosclerosis 1992; 97(2-3): 171-85.

[17] Cagatay P, Susleyici-Duman B, Ciftci C. Lipoprotein lipase gene PvuII polymorphism serum lipids and risk for coronary artery disease: meta-analysis. Dis Markers 2007; 23(3): 161-6.

[18] Fox CS, Massaro JM, Hoffmann U, et al. Abdominal visceral and subcutaneous adipose tissue compartments: association with metabolic risk factors in the Framingham Heart Study. Circulation 2007; 116(1): 39-48.

[19] Sumner AE, Vega GL, Genovese DJ, et al. Normal triglyceride levels despite insulin resistance in African Americans: role of lipoprotein lipase. Metabolism 2005; 54(7): 902-9.

[20] Stein E, Kushner H, Gidding S, et al. Plasma lipid concentrations in nondiabetic African American adults: associations with insulin resistance and the metabolic syndrome. Metabolism 2007; 56(7): 954-60.
[21] Trikalinos TA, Salanti G, Khoury MJ, et al. Impact of violations and deviations in Hardy-Weinberg equilibrium on postulated genedisease associations. Am J Epidemiol 2006; 163(4): 300-9.

[22] Lara-Castro C, Hunter GR, Lovejoy JC, et al. Apolipoprotein A-II polymorphism and visceral adiposity in African-American and white women. Obes Res 2005; 13(3): 507-12.

[23] Gower BA, Nagy TR, Goran MI, et al. Fat distribution and plasma lipid-lipoprotein concentrations in pre- and postmenopausal women. Int J Obes Relat Metab Disord 1998; 22(7): 605-11.

[24] Huffman DM, Landy NM, Potter E, et al. Comparison of the Lunar DPX-L and Prodigy dual-energy X-ray absorptimeters for assessing total and regional body composition. Int J Body Compos Res 2005; 3: 25-30.

[25] Kekes-Szabo T, Hunter GR, Nyikos I, et al. Development and validation of computed tomography derived anthropometric regression equations for estimating abdominal adipose tissue distribution. Obesity Res 1994; 2: 450-7.

[26] Goran M, Kaskoun MC, Shuman WP. Intra-abdominal adipose tissue in young children. Int J Obes 1995; 19: 279-83.

[27] Welch S, Gebhart SS, Bergman RN, et al. Minimal model analysis of intravenous glucose tolerance test-derived insulin sensitivity in diabetic subjects. J Clin Endocrinol Metab 1990; 71(6): 1508-18.

[28] Bergman RN, Phillips LS, Cobelli C. Physiologic evaluation of factors controlling glucose tolerance in man. Measurement of insulin sensitivity and beta-cell glucose sensitivity from the response to intravenous glucose. J Clin Invest 1981; 68: 1456-67.

[29] Pacini G, Bergman RN. MINMOD: a computer program to calculate insulin sensitivity and pancreatic responsivity from the frequently sampled intravenous glucose tolerance test. Comput Methods Programs Biomed 1986; 23: 113-22.

[30] Yang YJ, Youn JH, Bergman RN. Modified protocols improve insulin sensitivity estimation using the minimal model. Am J Physiol 1987; 253: E595-E602.

[31] Akey JM, Sosnoski D, Parra E, et al. Melting curve analysis of SNPs (McSNP): a gel-free and inexpensive approach for SNP genotyping. Biotechniques 2001; 30(2): 358-7.

[32] Parra EJ, Marcini A, Akey J, et al. Estimating African American admixture proportions by use of population-specific alleles. Am J Hum Genet 1998; 63(6): 1839-51.

[33] McKeigue PM. Multipoint admixture mapping. Genet Epidemiol 2000; 19(4): 464-7.

[34] Wen M, Christakis NA. Neighborhood effects on posthospitalization mortality: a population-based cohort study of the elderly in Chicago. Health Serv Res 2005; 40(4): 1108-27.

[35] Gower BA, Herd SL, Goran MI. Anti-lipolytic effects of insulin in African American and white prepubertal boys. Obes Res 2001; 9(3): 224-8.

[36] Carr DB, Utzschneider KM, Hull RL, et al. Intra-abdominal fat is a major determinant of the National Cholesterol Education Program Adult Treatment Panel III criteria for the metabolic syndrome. Diabetes 2004; 53(8): 2087-94.

[37] Despres JP, Couillard C, Gagnon J, et al. Race, visceral adipose tissue, plasma lipids, and lipoprotein lipase activity in men and women: the health, risk factors, exercise training, and genetics (HERITAGE) family study. Arterioscler Thromb Vasc Biol 2000; 20(8): 1932-8.

[38] Ferrannini E, Muscelli E, Natali A, et al. Association of fasting glucagon and proinsulin concentrations with insulin resistance. Diabetologia 2007; 50(11): 2342-7.

[39] Wajchenberg BL. Subcutaneous and visceral adipose tissue: their relation to the metabolic syndrome. Endocr Rev 2000; 21(6): 697738.

[40] Mori Y, Hoshino K, Yokota K, et al. Differences in the pathology of the metabolic syndrome with or without visceral fat accumulation: a study in pre-diabetic Japanese middle-aged men. Endocrine 2006; 29(1): 149-53.

[41] Howard BV, Cowan LD, Go O, et al. Adverse effects of diabetes on multiple cardiovascular disease risk factors in women. The Strong Heart Study. Diabetes Care 1998; 21(8): 1258-65.

[42] Fernandez JR, Allison DB. Understanding racial differences in obesity and metabolic syndrome traits. Nutr Rev 2003; 61(9): 3169 . 
[43] Gower BA, Fernandez JR, Beasley TM, et al. Using genetic admixture to explain racial differences in insulin-related phenotypes. Diabetes 2003; 52(4): 1047-51.

[44] Hall S, Chu G, Miller G, et al. A common mutation in the lipoprotein lipase gene promoter, $-93 \mathrm{~T} / \mathrm{G}$, is associated with lower plasma triglyceride levels and increased promoter activity in vitro. Arterioscler Thromb Vasc Biol 1997; 17/10: 1969-76.

[45] Salanti G, Higgins JP, Trikalinos TA, et al. Bayesian meta-analysis and meta-regression for gene-disease associations and deviations from Hardy-Weinberg equilibrium. Stat Med 2007; 26(3): 553-67.

Received: February 21, 2009

Revised: March 26, 2009

Accepted: March 30, 2009

(C) Casazza et al.; Licensee Bentham Open.

This is an open access article licensed under the terms of the Creative Commons Attribution Non-Commercial License (http: //creativecommons.org/licenses/bync/3.0/), which permits unrestricted, non-commercial use, distribution and reproduction in any medium, provided the work is properly cited. 